\title{
Cost analysis of office-based transnasal esophagoscopy
}

\author{
David J. Wellenstein ${ }^{1}$ (D . Jimmie Honings ${ }^{1} \cdot$ Henrieke W. Schutte ${ }^{1} \cdot$ Jasmijn M. Herruer ${ }^{1}$. \\ Frank J. A. van den Hoogen ${ }^{1} \cdot$ Henri A. M. Marres ${ }^{1} \cdot$ Robert P. Takes $^{1} \cdot$ Guido B. van den Broek ${ }^{1}$
}

Received: 2 January 2019 / Accepted: 20 February 2019 / Published online: 26 February 2019

(c) The Author(s) 2019

\begin{abstract}
Purpose Although office-based transnasal esophagoscopy has been investigated extensively, a cost analysis is still lacking. We performed a cost analysis combined with feasibility study for two diagnostic processes: patients with globus pharyngeus and/or dysphagia, and hypopharyngeal carcinoma.

Methods Prospective cohort study.

Results Forty-one procedures were performed, of which 35 were fully completed. The procedure was well tolerated with mild complaints such as nasal or pharyngeal pain and burping. Four complications occurred: two minor epistaxis and two vasovagal reactions. In patients with globus pharyngeus and/or dysphagia, transnasal esophagoscopy resulted in a cost saving of $€ 94.43$ ( $p$ 0.026) per procedure, compared to our regular diagnostic process. In patients with suspicion of hypopharyngeal carcinoma, cost savings were $€ 831.41$ ( $p 0.000)$ per case.

Conclusions Cost analysis showed that office-based transnasal esophagoscopy can provide significant cost savings for the current standard of care. Furthermore, this procedure resulted in good patient acceptability and few complications.
\end{abstract}

Keywords Transnasal esophagoscopy $\cdot$ Office-based $\cdot$ Topical anesthesia $\cdot$ Head and neck oncology $\cdot$ Cost analysis

\section{Introduction}

Inspection of the gastrointestinal tract with a flexible fiberoptic transoral endoscope became available in the late 1950s [1]. Shortly thereafter, Hirschowitz reported on a flexible fiberoptic esophagoscope with two working channels, enabling suction or obtaining biopsies [2]. Since the 1980s, fiberoptic endoscopy has slowly been replaced by distal chip endoscopy. In these endoscopes, a charge-coupled device (CCD) chip is located in the tip of the endoscope and images are seen on a video screen [3]. Since then, addition of a working channel in the digital endoscope and enhancement of image quality has transformed the field of diagnostic and therapeutic endoscopy. Through this ongoing development, endoscopes with smaller diameters became available, and thus the first studies were published on transnasal esophagoscopy (TNE) in the 1990s [4, 5].

David J. Wellenstein

david.wellenstein@radboudumc.nl

1 Department of Otorhinolaryngology and Head and Neck Surgery, Radboud University Medical Center, Philips van Leydenlaan 15, 6500 HB Nijmegen, The Netherlands
Since then, TNE has been extensively reported on, and has proved to accurately diagnose esophageal pathology [6-16]. Several studies showed better patient acceptability and less cardiopulmonary stress (i.e., rise in blood pressure and heart rate) during TNE, compared to transoral esophagoscopy [4, 15, 17-29]. Furthermore, TNE can be used for therapeutic office-based procedures under topical anesthesia, such as foreign body removal or esophageal balloon dilatation [30].

For otorhinolaryngologists and head and neck surgeons, inspection of the esophagus can be useful, in patients suffering from globus pharyngeus or dysphagia, to directly exclude mucosal esophageal pathology. Traditional diagnostics for these patients are limited to flexible pharyngolaryngoscopy, video fluoroscopy, rigid esophagoscopy under general anesthesia, and referral to a gastroenterologist in cases requiring flexible esophageal inspection. Furthermore, in the diagnostic workup of hypopharyngeal carcinoma, inspection of the proximal esophagus is usually performed under general anesthesia to determine the distal border of the tumor. With the introduction of TNE, examination of the esophagus for these indications can be performed in the outpatient clinic 
under topical anesthesia and additional indications are likely to be developed and established in the future [30, 31].

Although TNE has been extensively investigated in the last 2 decades, a cost analysis is still awaited [32]. There are several articles that reported on cost savings of TNE under topical anesthesia, but most were estimated cost savings [11, $18,33]$. Therefore, the goal of this study was to determine the actual cost difference for office-based TNE compared to two regular diagnostic processes. In patients with globus pharyngeus and/or dysphagia, the costs of TNE under topical anesthesia were compared with traditional flexible laryngoscopy and video fluoroscopy. In patients suspected of hypopharyngeal carcinoma, costs of TNE under topical anesthesia including taking biopsies were compared to the common practice of inspection under general anesthesia including biopsies. Furthermore, patient experiences and safety were evaluated.

\section{Materials and methods}

\section{Patient inclusion}

This prospective study was conducted in accordance with the guidelines established in the Declaration of Helsinki and was approved by the local medical ethical committee of our center (2015-2156). We estimated that 35 completed procedures would be sufficient to perform a cost analysis and investigate feasibility of office-based TNE. The first included patient category were adult patients with globus pharyngeus and/or dysphagia. All patients underwent a complete examination, including laryngoscopy, in our hospital or in the hospital from which they were referred. This patient category usually already underwent regular diagnostics (e.g., video fluoroscopy) and therapy (e.g., proton pump inhibitor or consultation of a speech language pathologist). Thus, they were examined to exclude a laryngopharyngeal or esophageal malignancy as the cause for their complaints. The second included patient category were patients with suspicion of hypopharyngeal carcinoma. These patients were referred from other (i.e., secondary) hospitals, if a suspicious tumor was seen during flexible laryngoscopy. Other hospitals refer patients immediately to reduce diagnostic delay. These patients were examined to identify the tumor, determine the distal extension in relation to the esophagus, and obtain biopsies under topical anesthesia the same day. Furthermore, they also underwent imaging diagnostics according to the national guidelines. There were no exclusion criteria.

\section{Setting}

This study was conducted in our tertiary referral center. Patients indicated for one of the two categories were included from January to September 2016. All patients were consecutively included, until 35 completed procedures were performed. This resulted in 41 attempted procedures.

\section{Costs data extraction}

Cost analysis was performed from a clinical diagnostic perspective, thus secondary costs (e.g., travel expenses, time off from work for patients undergoing the diagnostic process and family) and capital expenditure were not accounted for. For each of the 35 patients that underwent TNE, costs in Euros for all materials and procedures were obtained. All used materials, prices and sources are displayed in Table 1 . Costs for the one-time purchase of a transnasal esophagoscope, transnasal laryngoscope and video processor, were depreciated in 5 years on an average of 50 procedures performed each year. The 35 patients were divided into two groups, the first group consisted of patients with globus pharyngeus and dysphagia $(n=20)$, and the second group consisted of patients with suspicion of hypopharyngeal carcinoma $(n=15)$.

For these two groups, the same number of consecutive patients that underwent the diagnostic process in the months before we started with TNE (i.e., the regular diagnostic process) were searched in our electronic health record system based on their medical record codes. For 20 patients with globus pharyngeus or dysphagia, the entire diagnostic process was evaluated and all costs were extracted. These patients underwent several outpatient clinic visits with laryngoscopy (VNL 1070STK, Pentax Medical, Uithoorn, The Netherlands), and if indicated video fluoroscopy (i.e., barium swallow examination). For the 15 patients with a suspicion of hypopharyngeal carcinoma, the diagnostic process before TNE was a consultation in the outpatient clinic with laryngoscopy, and afterwards an investigation (laryngopharyngoscopy and proximal esophagoscopy) under general anesthesia with biopsies and daycare admission (no overnight stay) to the inpatient ward. The average number of procedures per patient category is displayed in Table 2 .

\section{TNE procedure}

Patients were examined in the outpatient clinic of our center. Elaborate patient instructions on topical anesthesia administration and the procedure were provided. The patient was seated, and topical nasal anesthesia was administered by placing 2-3 gauze pledgets soaked in $10 \%$ lidocaine and $0.1 \%$ xylometazoline in each nasal cavity. The gauze pledgets were left in place for a minimum of 10-15 min. Furthermore, laryngopharyngeal anesthesia was administered by applying around ten sprays of $10 \%$ lidocaine, which is lower than the maximum dose of lidocaine application in the larynx [34]. Also, the tip of the 
Table 1 Costs for each material per patient category

\begin{tabular}{|c|c|c|c|}
\hline Parameter & TNE $(€)$ & $\begin{array}{l}\text { Regular diagnostic process for globus } \\
\text { pharyngeus and/or dysphagia }(€)\end{array}$ & $\begin{array}{l}\text { Regular diagnostic process for } \\
\text { hypopharyngeal carcinoma }(€)\end{array}$ \\
\hline Lidocaine & $0.18^{\mathrm{a}}$ & & \\
\hline Attachment for spraying & $0.82^{\mathrm{a}}$ & & \\
\hline Xylometazoline & $1.02^{\mathrm{a}}$ & & \\
\hline Gauze pledgets (10 units) & $0.07^{\mathrm{a}}$ & & \\
\hline Biopsy forceps & $17.00^{\mathrm{a}}$ & & \\
\hline Pathology container & $1.29^{\mathrm{a}}$ & & $1.29^{\mathrm{a}}$ \\
\hline Single wash of endoscope & $24.00^{\mathrm{a}}$ & & \\
\hline Video fluoroscopy & & $281.26^{\mathrm{a}}$ & \\
\hline Consulting pathologist & $114.38^{\mathrm{a}}$ & & $114.38^{\mathrm{a}}$ \\
\hline Surgery (half hour) & & & $440.00^{\mathrm{a}}$ \\
\hline 1-day ward administration & & & $476.00^{\mathrm{b}}$ \\
\hline Outpatient clinic visit & $91.00^{\mathrm{b}}$ & $91.00^{\mathrm{b}}$ & $91.00^{\mathrm{b}}$ \\
\hline Single-use transnasal esophagoscope & $98.87^{\mathrm{c}}$ & & \\
\hline Single-use video processor & $94.68^{\mathrm{d}}$ & $94.68^{\mathrm{d}}$ & $94.68^{\mathrm{d}}$ \\
\hline Single-use transnasal laryngoscope & $85.12^{\mathrm{e}}$ & $85.12^{\mathrm{e}}$ & $85.12^{\mathrm{e}}$ \\
\hline
\end{tabular}

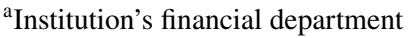

${ }^{b}$ Dutch Health Institute. Guideline for performance of economic evaluations in healthcare. February 29, 2016 version. https://www.zorginstit uutnederland.nl/over-ons/publicaties/publicatie/2016/02/29/richtlijn-voor-het-uitvoeren-van-economische-evaluaties-in-de-gezondheidszorg (in Dutch). Accessed February 16, 2018

${ }^{c}$ Pentax Medical; $€ 24,718.00 / 5$ (years)/50 (patient's per year)

${ }^{\mathrm{d}}$ Pentax Medical; €23,669.00/5 (years)/50 (patient's per year)

${ }^{\mathrm{e}}$ Pentax Medical; $€ 21.281 .00 / 5$ (years)/50 (patient's per year)

Table 2 Average number of products used and procedures performed per patient category

\begin{tabular}{lllll}
\hline Parameter & TNE (G/D) & TNE $(\mathrm{H})$ & $\begin{array}{l}\text { Regular diagnostic } \\
\text { process }(\mathrm{G} / \mathrm{D})\end{array}$ & $\begin{array}{l}\text { Regular diag- } \\
\text { nostic process } \\
(\mathrm{H})\end{array}$ \\
\hline Lidocaine & 1 & 1 & 0 & 0 \\
Attachment for spraying & 1 & 1 & 0 & 0 \\
Xylometazoline & 1 & 1 & 0 & 0 \\
Gauze pledgets (10 units) & 1 & 1 & 0 & 0 \\
Biopsy forceps & 0.35 & 0.73 & 0 & 0 \\
Pathology container & 0.35 & 0.73 & 0 & 1 \\
Single wash of endoscope & 1 & 1 & 0 & 0 \\
Video fluoroscopy & 0 & 0 & 0.8 & 0.07 \\
Consulting pathologist & 0.35 & 0.35 & 0 & 1 \\
Surgery (half hour) & 0 & 0 & 0 & 1 \\
1-day ward administration & 0 & 0 & 0 & 1 \\
Outpatient clinic visit & 1 & 2 & 2.35 & 2.05 \\
Transnasal esophagoscopy & 1 & 1 & 0 & 0 \\
Transnasal laryngoscopy & 1 & 1 & 1.55 & 1.05 \\
\hline
\end{tabular}

$G / D$ globus pharyngeus and/or dysphagia, $H$ hypopharyngeal carcinoma endoscope was lubricated with lidocaine gel. Patients were advised not to eat or drink until $1 \mathrm{~h}$ after the last laryngopharyngeal anesthesia administration, to avoid aspiration due to a desensitized laryngopharynx.
For TNE, a transnasal esophagoscope was used (EE1580K, Pentax Medical, Uithoorn, The Netherlands). This endoscope has a $5.1 \mathrm{~mm}$ outer diameter with a $2.0 \mathrm{~mm}$ working channel, allowing suction or insufflation during 
examination. Digital images were processed using a video processor (EPK-i5000-HD, Pentax Medical, Uithoorn, The Netherlands).

After pharyngolaryngoscopy, the endoscope was directed into the pyriform sinus. The patient was asked to swallow, after which the endoscope was inserted into the esophagus and passed into the stomach. The stomach was inspected, including the caudal portion of the distal esophageal sphincter, by retroflexion of the tip of the endoscope, also known as the 'J-maneuver'. Inspection of the mucosa of the esophagus was performed by retracting the endoscope slowly upwards, gaining circumferential sight of the esophagus by repeated insufflations of air through the working channel of the endoscope. In case of suspicious lesions, biopsies were taken with a flexible endoscopic biopsy forceps (Radial Jaw ${ }^{\mathrm{TM}} 4$ pulmonary standard capacity with needle $1.8 \mathrm{~mm}$ diameter, Boston Scientific, Costa Rica).

After TNE, patients were asked to complete a questionnaire containing five questions regarding their experiences during the procedure. A visual analogue scale (VAS) was used to rate patients' experiences concerning nasal pain during endoscope insertion, throat pain during examination, and inconvenience due to gag reflex, nausea, and burping. Each question is rated on a scale from 1 to 10 , where 1 is the least unpleasant and 10 is the most unpleasant.

\section{Analysis}

Statistical analysis was performed using IBM Statistical Package for Social Sciences Statistics 22 (IBM Corp. Released 2013. IBM SPSS Statistics for Windows, Version 22.0. Armonk, NY: IBM Corp). For cost analysis, the two groups were analyzed using independent-sample $t$ test and bootstrapping.

\section{Results}

Between January 2016 and September 2016, 41 TNE procedures under topical anesthesia were attempted. The results are summarized in Table 3. Cost analysis for the first group (i.e., patients with globus pharyngeus and/or dysphagia) revealed a significant cost difference in favor of TNE. The mean difference in costs was $€ 94.43$ ( $p$ 0.026) per procedure, with mean costs of $€ 532.80$ for TNE and $€ 627.23$ for the regular diagnostic process. This difference remained statistically significant after bootstrapping ( $p$ 0.035). For the second group (i.e., patients with suspicion of hypopharyngeal carcinoma), even more extensive differences were found. The diagnostic process with office-based TNE (mean costs $€ 583.54$ ) was significantly less expensive ( $p$ 0.000) compared to the regular diagnostic process (mean costs $€ 1414.95$ ), with a mean cost difference of $€ 831.41$ per procedure. Again, the difference remained statistically significant after bootstrapping ( $p 0.001)$.

Thirty-five procedures $(85.3 \%)$ were completed. Mean VAS score for patient experience was 2.2 for burping, 1.9 for nasal pain during insertion, 1.7 for throat pain, 1.5 for gagging, and 0.3 for nausea. Six procedures were not completed, four $(9.8 \%)$ due to failure of passage of the esophagoscope through the nose, and two (4.9\%) due to vasovagal reaction of the patient. Both patients experienced light headedness and nausea, but did not lose consciousness, and recovered without sequelae. Two patients experienced epistaxis after TNE, which was resolved after placing cotton pledgets soaked in $0.1 \%$ xylometazoline in the nasal cavity. After a short observation, both patients fully recovered without sequelae. Of the 20 patients that underwent TNE for globus pharyngeus and/or dysphagia, 4 cases (20\%) revealed primary or residual laryngeal carcinoma and for 4 cases $(20 \%)$ primary esophageal carcinoma.

\section{Discussion}

Although office-based TNE has been extensively investigated in the last decades and several studies mentioned the estimated cost savings for office-based TNE, a cost analysis was never performed $[11,18]$. Therefore, we conducted this prospective clinical study to analyze the cost savings of TNE for two diagnostic indications, and investigate the feasibility at our tertiary referral center.

We demonstrated that office-based TNE provides significant cost savings in patients suffering from globus pharyngeus and/or dysphagia, in whom the primary goal was to exclude an esophageal tumor as the cause of their complaints. Even more significant cost reduction was found in patients with (suspicion of) hypopharyngeal carcinoma that underwent office-based TNE with biopsies. In these patients, examination under general anesthesia with biopsies can be omitted. As was expected, cost savings were even greater compared to cost savings for patients with globus pharyngeus and dysphagia.

An overall advantage of TNE is the favorable patient acceptance, as our own experience and several other studies have shown [30]. Only few minor complications occurred, with no long-lasting consequences for the patient. When reviewing our complications compared to the literature, we noticed higher rates of epistaxis and vasovagal reaction [30]. In patients with globus pharyngeus and/or dysphagia, 20\% of the patients had esophageal carcinoma found during TNE. Although this rate was surprisingly high, similar rates have been reported in the literature [30]. Patients with globus pharyngeus and/or dysphagia that are referred to our tertiary hospital, are probably a selected group of patients and different, compared to the category of patients that are seen 
Table 3 Patient characteristics

\begin{tabular}{|c|c|c|}
\hline Characteristics & TNE & $\%$ \\
\hline Study population & 41 & 100 \\
\hline Sex (males) & 28 & 68.3 \\
\hline Age (range) & $66.6(29-87)$ & \\
\hline \multicolumn{3}{|l|}{ Indication } \\
\hline Globus pharyngeus and/or dysphagia & 26 & 63.4 \\
\hline Suspicion hypopharyngeal/esophageal carcinoma & 15 & 36.6 \\
\hline Completed procedures & 35 & 85.3 \\
\hline Discontinued procedures & 6 & 14.7 \\
\hline No nasal passage endoscope & 4 & 9.8 \\
\hline Complication & 2 & 4.9 \\
\hline Duration (range minutes) & $15.03(6.38-35.00)$ & \\
\hline Clinical findings & 35 & 100 \\
\hline Globus pharyngeus and/or dysphagia & 20 & 57.1 \\
\hline No suspicious lesions & 11 & 31.4 \\
\hline Laryngeal cyst & 2 & 5.7 \\
\hline Barrett's esophagus $^{\mathrm{a}}$ & 1 & 2.9 \\
\hline Laryngeal carcinoma (primary or residual) & 4 & 11.4 \\
\hline Esophageal carcinoma ${ }^{\mathrm{b}}$ & 4 & 11.4 \\
\hline Suspected hypopharyngeal tumor & 10 & 28.6 \\
\hline Hypopharyngeal carcinoma & 8 & 22.9 \\
\hline No suspicious lesions & 2 & 5.7 \\
\hline Suspected esophageal tumor ${ }^{\mathrm{c}}$ & 5 & 14.3 \\
\hline Esophageal carcinoma & 1 & 2.9 \\
\hline No suspicious lesions & 4 & $11.4(4 / 35)$ \\
\hline Laryngeal carcinoma $^{\mathrm{d}}$ & 1 & 2.9 \\
\hline \multicolumn{3}{|l|}{ Complication } \\
\hline Epistaxis & 2 & 4.9 \\
\hline Vasovagal reaction & 2 & 4.9 \\
\hline \multicolumn{3}{|l|}{ VAS score patient tolerance (average) ${ }^{\mathrm{e}}$} \\
\hline Burping & 2.2 & \\
\hline Pain in nose & 1.9 & \\
\hline Pain in throat & 1.7 & \\
\hline Gagging & 1.5 & \\
\hline Nausea & 0.3 & \\
\hline
\end{tabular}

${ }^{a}$ One patient with globus pharyngeus and dysphagia had residual laryngeal carcinoma and Barrett's esophagus

${ }^{\mathrm{b}}$ One patient with globus pharyngeus and dysphagia had a tumor in the oral cavity, oropharynx and esophagus

${ }^{\mathrm{c}}$ These were patients with suspected lesions that were seen on PET and/or CT

${ }^{\mathrm{d}}$ One patient with a suspected esophageal tumor on PET showed no pathology in the esophagus, but residual laryngeal carcinoma

${ }^{\mathrm{e}}$ Visual analogue scale (VAS): $0=$ no complaints, $10=$ unbearable complaints

in a non-academic secondary referral clinic. Most esophageal pathologies were found in patients suffering from both globus pharyngeus and dysphagia, thus this combination might be a strong indication to perform TNE. An ongoing point of discussion is the screening for second primary tumors in the esophagus by esophagoscopy in patients with hypopharyngeal carcinoma, given the relatively higher incidence of esophageal carcinoma in these patients [30]. The short duration of a TNE, with few complications and good patient acceptance, could favor performing TNE, especially if the incidence rates of esophageal carcinoma are as high as found in our study.

Although we did not investigate this in our current study, our experience is that office-based TNE results in a faster diagnostic process [35]. In patients with globus pharyngeus and/or dysphagia, office-based TNE gives 
less burden for the patient. When no pathology is found, patients can be reassured immediately and secondary diagnostics (e.g., video fluoroscopy or referral to a gastroenterologist) can be avoided. Furthermore, TNE might replace video fluoroscopy, as it is a faster procedure, with significant cost savings and no radiation exposure. Due to this study, a significant decrease in video fluoroscopy has occurred in our center for patients with globus pharyngeus and/or dysphagia. If esophageal pathology is encountered during endoscopy, histology can also be obtained. All patients referred to us with suspicion of a head and neck malignancy are seen weekly in our multidisciplinary head and neck oncological center. With the introduction of TNE in the diagnostic process, patients with suspected hypopharyngeal carcinoma undergo TNE the same day to identify the distal border of the tumor, and biopsies are obtained. By arranging a fast diagnostic track, biopsies during TNE (instead of endoscopy under general anesthesia) are evaluated within 2 days by the pathologist and our diagnostic process has shortened from two and a half weeks, to 2 days.

Potential limitations of this study are the limited number of patients included and the lack of a power analysis, which could result in less reliable data. We chose the number of 35 participants in the context of evaluation of feasibility for TNE at our department. We performed a post hoc power analysis, that showed high power $(1-\beta 0.91)$ for the chosen sample size. Furthermore, indirect costs (i.e., secondary costs and capital expenditure) were not evaluated in this study, because our goal was to evaluate the cost savings from a medical perspective. By including these costs, such as travel time and time of absence from work of patients and their family, a more robust cost analysis could be performed.

In conclusion, office-based TNE resulted in significant cost savings, for patients with globus pharyngeus and/or dysphagia, and suspicion of hypopharyngeal carcinoma. Furthermore, this procedure resulted in good patient acceptance and had few complications.

\section{Compliance with ethical standards}

Conflict of interest This study was supported by an unrestricted educational grant from Pentax Medical Europe.

Ethical approval All procedures performed in studies involving human participants were in accordance with the ethical standards of the institutional and/or national research committee and with the 1983 Helsinki Declaration and its later amendments or comparable ethical standards.

Informed consent Informed consent was obtained from all individual participants included in the study.
Open Access This article is distributed under the terms of the Creative Commons Attribution 4.0 International License (http://creativeco mmons.org/licenses/by/4.0/), which permits unrestricted use, distribution, and reproduction in any medium, provided you give appropriate credit to the original author(s) and the source, provide a link to the Creative Commons license, and indicate if changes were made.

\section{References}

1. Hirschowitz BI, Peters CW, Curtiss LE (1957) Preliminary report on a long fiberscope for examination of stomach and duodenum. Med Bull (Ann Arbor) 23(5):178-180

2. Hirschowitz BI (1963) A fibre optic flexible cesophagoscope. Lancet 2(7304):388

3. Marsh BR (1996) Historic development of bronchoesophagology. Otolaryngol Head Neck Surg 114(6):689-716

4. Shaker R (1994) Unsedated trans-nasal pharyngoesophagogastroduodenoscopy (T-EGD): technique. Gastrointest Endosc 40(3):346-348

5. Herrmann IF, Recio SA (1997) Laryngeal and tracheal surgery functional pharyngoesophagoscopy: a new technique for diagnostics and analyzing deglutition. Oper Tech Otolaryngol Head Neck Surg 8(3):163-167

6. Aviv JE, Takoudes TG, Ma G, Close LG (2001) Office-based esophagoscopy: a preliminary report. Otolaryngol Head Neck Surg 125(3):170-175

7. Belafsky PC, Postma GN, Daniel E, Koufman JA (2001) Transnasal esophagoscopy. Otolaryngol Head Neck Surg 125(6):588-589

8. Postma GN, Bach KK, Belafsky PC, Koufman JA (2002) The role of transnasal esophagoscopy in head and neck oncology. Laryngoscope 112(12):2242-2243

9. Andrus JG, Dolan RW, Anderson TD (2005) Transnasal esophagoscopy: a high-yield diagnostic tool. Laryngoscope 115(6):993-996

10. Postma GN, Cohen JT, Belafsky PC et al (2005) Transnasal esophagoscopy: revisited (over 700 consecutive cases). Laryngoscope 115(2):321-323

11. Price T, Sharma A, Snelling J et al (2005) How we do it: the role of trans-nasal flexible laryngo-oesophagoscopy (TNFLO) in ENT: one year's experience in a head and neck orientated practice in the UK. Clin Otolaryngol 30(6):551-556

12. Bennett AM, Sharma A, Price T, Montgomery PQ (2008) The management of foreign bodies in the pharynx and oesophagus using transnasal flexible laryngo-oesophagoscopy (TNFLO). Ann R Coll Surg Engl 90(1):13-16

13. Cheng CC, Fang TJ, Lee TJ et al (2012) Role of flexible transnasal esophagoscopy and patient education in the management of globus pharyngeus. J Formos Med Assoc 111(3):171-175

14. Dolan RW, Anderson TD (2013) Practical applications of in-office fiberoptic transnasal esophagoscopy in the initial evaluation of patients with squamous cell cancer of the head and neck. Ear Nose Throat J 92(9):450-455

15. Chung EJ, Rho YS, Jung KY, Kim JW, Lee SW (2014) The role of transnasal esophagoscopy in ENT office: a prospective, multicenter study in Korea. Clin Exp Otorhinolaryngol 7(2):123-125

16. Abou-Nader L, Wilson JA, Paleri V (2014) Transnasal oesophagoscopy: diagnostic and management outcomes in a prospective cohort of 257 consecutive cases and practice implications. Clin Otolaryngol 39(2):108-113

17. Campo R, Montserrat A, Brullet E (1998) Transnasal gastroscopy compared to conventional gastroscopy: a randomized study of feasibility, safety, and tolerance. Endoscopy 30(5):448-452 
18. McPartlin DW, Nouraei SA, Tatla T, Howard DJ, Sandhu GS (2005) How we do it: transnasal fibreoptic oesophagoscopy. Clin Otolaryngol 30(6):547-550

19. Sharma A, Price T, Mierzwa K, Montgomery P, Qayyum A, Bradnam T (2006) Transnasal flexible laryngo-oesophagoscopy: an evaluation of the patient's experience. J Laryngol Otol 120(1):24-31

20. Chang JY, Talley NJ, Locke GR 3rd et al (2011) Population screening for barrett esophagus: a prospective randomized pilot study. Mayo Clin Proc 86(12):1174-1180

21. Kataoka H, Hayano J, Mizushima T et al (2011) Cardiovascular tolerance and autonomic nervous responses in unsedated upper gastrointestinal small-caliber endoscopy: a comparison between transnasal and peroral procedures with newly developed mouthpiece. Dig Endosc 23(1):78-85

22. Ai ZL, Lan CH, Fan LL et al (2012) Unsedated transnasal upper gastrointestinal endoscopy has favorable diagnostic effectiveness, cardiopulmonary safety, and patient satisfaction compared with conventional or sedated endoscopy. Surg Endosc 26(12):3565-3572

23. Chung JW, Park S, Chung MJ et al (2012) A novel disposable, transnasal esophagoscope: a pilot trial of feasibility, safety, and tolerance. Endoscopy 44(2):206-209

24. Knuth J, Kunze DE, Benz C et al (2013) Is the transnasal access for esophagogastroduodenoscopy in routine use equal to the transoral route? A prospective, randomized trial. Z Gastroenterol 51(12):1369-1376

25. Kadayifci A, Atar M, Parlar S, Balkan A, Koruk I, Koruk M (2014) Transnasal endoscopy is preferred by transoral endoscopy experienced patients. J Gastrointestin Liver Dis 23(1):27-31

26. Streckfuss A, Bosch N, Plinkert PK, Baumann I (2014) Transnasal flexible esophagoscopy (TNE): an evaluation of the patient's experience and time management. Eur Arch Otorhinolaryngol 271(2):323-328

27. Young VN, Smith LJ, Sulica L, Krishna P, Rosen CA (2012) Patient tolerance of awake, in-office laryngeal procedures: a multiinstitutional perspective. Laryngoscope 122(2):315-321
28. Dumortier J, Napoleon B, Hedelius F et al (2003) Unsedated transnasal EGD in daily practice: results with 1100 consecutive patients. Gastrointest Endosc 57(2):198-204

29. Tsuboi M, Arai M, Maruoka D et al (2013) Utility and stability of transnasal endoscopy for examination of the pharynx - a prospective study and comparison with transoral endoscopy. Int J Med Sci 10(9):1085-1091

30. Wellenstein DJ, Schutte HW, Marres HAM et al (2017) Officebased procedures for diagnosis and treatment of esophageal pathology. Head Neck Sep;39(9):1910-1919

31. Howell RJ, Pate MB, Ishman SL, Isseroff TF, Rubin AD, Soliman AM et al (2016) Prospective multi-institutional transnasal esophagoscopy: predictors of a change in management. Laryngoscope 126(12):2667-2671

32. Sabirin J, Abd Rahman M, Rajan P (2013) Changing trends in oesophageal endoscopy: a systematic review of transnasal oesophagoscopy. ISRN Otolaryngol 2013:586973

33. Bush CM, Postma GN (2013) Transnasal esophagoscopy. Otolaryngol Clin N Am 46(1):41-52

34. Wellenstein DJ, van der Wal RAB, Schutte HW et al (2018) Topical anesthesia for endoscopic office-based procedures of the upper aerodigestive tract. J Voice. https://doi.org/10.1016/j.jvoic e.2018.02.006

35. Schutte HW, Slootweg PJ, Takes RP et al (2018 Nov) Digital video laryngoscopy and flexible endoscopic biopsies as an alternative diagnostic work-up in laryngopharyngeal cancer; a prospective clinical study. Ann Otol Rhinol Laryngol 127(11):770-776

Publisher's Note Springer Nature remains neutral with regard to jurisdictional claims in published maps and institutional affiliations. 